\title{
Deposition of Cohesive Sediment from Turbulent Plumes, Gravity Currents, and Turbidity Currents
}

\author{
Gregory F. Lane-Serff, Ph.D. ${ }^{1}$
}

\begin{abstract}
Models for the deposition of cohesive sediment from turbulent plumes (or "buoyant jets"), gravity currents, and turbidity currents are provided in this paper. The cohesive sediment is made up of small particles that aggregate together to form larger flocs, which are in turn broken up by turbulent shear. The equilibrium mean floc size (and thus the equilibrium mean fall speed) is a function of the turbulent dissipation rate and the sediment concentration. The flows are modeled by using integral and box models, with dissipation related to bulk flow properties. For plumes it is shown that there is a well-defined equilibrium fall speed at the virtual origin and that the fall speed changes relatively slowly in the momentum-dominated part of the flow (within one jet length or so of the source). If the flocs are assumed to adjust instantaneously to their equilibrium size, an integral model for a turbulent plume carrying cohesive sediment can be described in terms of two parameters: the angle between the plume and the horizontal at the virtual origin and the (nondimensional) fall speed there. Next, a typical time scale for flocs to adjust to their equilibrium size is identified, and the model is extended to include an equation for the rate of change of the mean floc size along the plume. The time scale over which the mean floc size changes can be compared with a natural time scale for the plume (the time taken for a particle traveling at the mean plume speed to travel a jet length). Thus, in this nonequilibrium model, a further nondimensional parameter is identified, $B$, which is proportional to the ratio of a typical plume time scale to the typical floc size adjustment time scale. When $B$ is large, the flocs adjust almost instantaneously to the equilibrium size, whereas when $B$ is very small, the flocs remain close to their size at the source. However, whatever the value of $B$ (which is in terms of typical time scales), the local adjustment time scale always tends to zero approaching an idealized source (virtual origin) so that the equilibrium model is always valid there. For plumes injected horizontally, the equilibrium floc size tends to reduce with distance from the source, with any reduction in turbulent shear more than compensated for by the reduction in sediment concentration. The equilibrium model is then applied to two-dimensional and axisymmetric gravity currents and turbidity currents. The gravity currents are assumed to be steady flows driven by a constant source of dense fluid with the sediment having a negligible effect on the fluid density. In contrast, the turbidity currents modeled are initiated by the release of a finite volume of fluid containing the sediment, with the sediment concentration providing the density difference from the ambient fluid. For these flows, the basic scales are identified, and the concentration and deposition distributions given. DOI: 10.1061/(ASCE)HY.1943-7900.0000463. ( 2011 American Society of Civil Engineers.
\end{abstract}

CE Database subject headings: Buoyancy; Plumes; Sediment; Outfall sewers.

Author keywords: Buoyant jets; Plumes; Sedimentation; Sediment discharge; Cohesive sediment; Outfall sewers; Sediment deposits.

\section{Introduction}

A range of buoyancy-driven flows in the natural and built environment contain particles that fall out of the main flow. In some cases, the particles aggregate together to form larger "flocs," which generally have a larger fall speed than the original individual component particles. Examples include flows containing muds or sewage sludges. Modeling the detailed flocculation process is complex because a range of factors connected with the individual particles (surface physics and chemistry) and with the turbulent flow must be considered. A description of such a model is given in Winterwerp (1998), and a more general review of flocculation models is given by Thomas et al. (1999). The process is dynamic, with the floc size a result of a competition between aggregating processes, which

${ }^{1}$ Senior Lecturer, School of Mechanical, Aerospace and Civil Engineering, Univ. of Manchester, Pariser Building, Sackville St., Manchester M13 9PL, UK. E-mail: g.f.lane-serff@manchester.ac.uk

Note. This manuscript was submitted on August 26, 2010; approved on May 20, 2011; published online on May 23, 2011. Discussion period open until May 1, 2012; separate discussions must be submitted for individual papers. This paper is part of the Journal of Hydraulic Engineering, Vol. 137, No. 12, December 1, 2011. CASCE, ISSN 0733-9429/2011/ $12-1615-1623 / \$ 25.00$. depend on the turbulence and the concentration of particles, and break-up processes, in which larger flocs are destroyed by turbulent shear. If the conditions are sufficiently (statistically) steady, then an equilibrium mean floc size can be found, where the two competing processes operate at the same rate.

This paper does not cover detailed modeling of flocculation. Instead it concentrates on results in terms of an established relation of equilibrium floc size (and fall speed) to bulk flow properties. To do this, the fall speed ( $W_{S 0}$, say) is assumed to be known for some standard flow conditions and expressions are found giving the equilibrium fall speed for other flow conditions relative to this standard. An approximate equation is also found that describes the rate at which the floc size tends toward the local equilibrium value. The floc model is applied to sedimentation from turbulent buoyant plumes by using an integral model.

In the next section, the model for cohesive sediment is described, and the main results are derived in terms of fall speed as a function of bulk flow properties, including the time scale for the flocculation/break-up process. This model is then applied to turbulent plumes (or "buoyant jets") injected at an angle to the vertical. After recapping integral models of plumes with noncohesive sediment, a simple equilibrium model for the cohesive sediment is applied, assuming that the flocs adjust instantaneously 
to their equilibrium size. The turbulent plume model is extended to include a nonequilibrium model for the mean floc size, where the rate at which flocs adjust to the equilibrium size is included. Next, the simple equilibrium model is applied to particle-laden gravity currents and turbidity currents. Finally, the main results are discussed, and some conclusions about modeling cohesive sediment are drawn.

\section{Modeling Cohesive Sediment}

\section{Equilibrium Floc Size}

This section first presents a brief summary and simplified version of the model for cohesive sediment presented by Winterwerp (1998), to which the reader is referred for a more detailed explanation (hereafter referred to as W98).

The size of the small, individual sediment particles is denoted by $D_{P}$, and the floc size is denoted by $D$. The flocs are assumed to be made up of many small particles, so that $D \gg D_{P}$. The turbulence is represented by a dissipation parameter, $G$, with

$$
G=\sqrt{\varepsilon / \nu}
$$

where $\varepsilon=$ rate of turbulent dissipation; and $\nu=$ fluid viscosity. Thus $G$ is proportional to the turbulent strain, and $1 / G$ is a measure of the Kolmogorov time scale, and the Kolmogorov length scale $\lambda=\nu^{1 / 2} G^{-1 / 2}$. The flocs are assumed to have a self-similar form, described by a fractal dimension denoted by $n_{f}$. The evolution of the typical floc size is found to be given by (see W98)

$$
\frac{d D}{d t}=k_{A} C G D^{4-n_{f}}-k_{B} G^{q+1}\left(D-D_{P}\right)^{p} D^{2 q+1}
$$

where $C=$ sediment concentration; $k_{A}=$ constant related to the aggregation process; $k_{B}=$ constant related to break up; and $p$ and $q=$ further model constants. W98 finds model constants for a riverine mud of $k_{A}=14.6 \mathrm{~m}^{2} \mathrm{~kg}^{-1}$ and $k_{B}=14.0 \times 10^{3} \mathrm{~s}^{1 / 2} \mathrm{~m}^{-2}$. The aggregation process, described by the first term on the right side of Eq. (2a), depends on both concentration (because higher concentrations lead to more collisions) and turbulent intensity (stronger turbulence also leads to more collisions). However, the break-up process, described by the second term on the right side of of Eq. (2a), depends on the turbulent intensity (stronger turbulence leads to increased turbulent shear) but not on the concentration. W98 further shows that the appropriate values for $p$ and $q$ are $\left(3-n_{f}\right)$ and $1 / 2$, respectively. This gives

$$
\frac{d D}{d t}=k_{A} C G D^{4-n_{f}}-k_{B} G^{3 / 2}\left(D-D_{P}\right)^{3-n_{f}} D^{2}
$$

At the equilibrium floc size, $D=D_{e}$, the two terms balance. In the next subsection, Eq. ( $2 b)$ is used to approximate the rate at which the flocs approach the equilibrium size. For muds, W98 finds a fractal dimension of $n_{f}=2$ and thus an equilibrium floc size given by

$$
D_{e}=D_{P}+\frac{k_{A} C}{k_{B} \sqrt{G}}
$$

Writing $g_{S}^{\prime}$ for $g\left(\rho_{S}-\rho\right) / \rho$, where $\rho_{S}=$ density of the sediment and $\rho=$ density of the fluid, gives a fall speed for flocs of equilibrium size (based on Stokes' law) of

$$
W_{S, e}=W_{S, P}+\alpha^{\prime} \frac{k_{A} g_{S}^{\prime}}{k_{B} \nu} D_{P} \frac{C}{\sqrt{G}}
$$

where $W_{S, P}=$ fall speed of individual particles; and $\alpha^{\prime}=$ constant. This paper further assumes that the flocs are much larger than the individual particles (i.e., $D_{e} \gg D_{P}$ ) and the fall speed is always close to the equilibrium value, so

$$
D_{e} \approx \frac{k_{A} C}{k_{B} \sqrt{G}} \quad \text { and } \quad W_{S} \approx W_{S, e} \propto D_{e}
$$

The result of this model is a prediction that the equilibrium floc size is related to the Kolmogorov length scale (because $\lambda \sim G^{1 / 2}$ ). This result is common to many flocculation models [see the reviews by Thomas et al. (1999) and Jarvis et al. (2005)] and is explained physically by the idea that flocs smaller than the Kolmogorov scale can only experience laminar flow, whereas larger flocs are exposed to the turbulent flow. Thus, although the focus here is on the W98 model, the results based on other models for equilibrium floc size would be similar.

It is important to note the dependence of $D_{e}$, and thus $W_{S}$, on $C$ and $G$ (which in turn depends on $\varepsilon$ ). In terms of these more basic parameters, we can write (for $n_{f}=2$ )

$$
W_{S} \propto \frac{C}{\varepsilon^{1 / 4}}
$$

For more general values of the fractal dimension, $n_{f}$, the functional dependence of the equilibrium floc size, $D_{e}$, is not affected by $n_{f}$ (at least for $D_{e} \gg D_{P}$ ), but the fall-speed relationship becomes

$$
W_{S} \propto\left(\frac{C}{\varepsilon^{1 / 4}}\right)^{n_{f}-1}
$$

Here the concentration is on the case described by Eq. ( $6 a)$, with $n_{f}=2$. However, it is relatively straightforward to use the more general version Eq. (6b) if appropriate, and this case is discussed in the final section.

\section{Nonequilibrium Floc Size}

If the time scale for floc aggregation and break up is very long, then the floc size (and fall speed) will remain at its initial value, and the behavior can be described by using the model for noncohesive sediment given by Lane-Serff and Moran (2005). On the other hand, if the time scale is relatively short, the flocs can be modeled as having the local equilibrium size and fall speed given by Eqs. (5) and (6a). In practice the flocs will generally lie somewhere between the initial values and the local equilibrium values.

Assuming that floc size is close to the local equilibrium value, then Eq. (2b) can be expanded about the equilibrium value to find

$$
\frac{d D^{\prime}}{d t} \approx-\left(\frac{k_{A}^{2} C^{2} G^{1 / 2}}{k_{B}}\right) D^{\prime}
$$

where $D^{\prime}=\left(D-D_{e}\right)=$ difference of the floc size from the local equilibrium value. Thus the floc size tends exponentially to the local equilibrium value on a time scale $T_{A}$ given by

$$
T_{A}=\frac{k_{B}}{k_{A}^{2} C^{2} G^{1 / 2}}
$$

Again, although this result is based on the W98 model, similar results would be expected from other models, with the floc size tending to the equilibrium size on a time scale similar to that in Eq. (7b). 


\section{Deposition from Buoyant Plumes}

In many practical applications (e.g., most marine outfalls) buoyant fluid is injected horizontally, but this work allows for any angle to the vertical. A description of an integral model for angled, buoyant plumes (in an unstratified ambient fluid) is given by Lane-Serff et al. (1993), and Lane-Serff and Moran (2005) extend this model to include (noncohesive) sediment. Further experimental work on deposition from plumes is given by Cuthbertson and Davies (2008) and Cuthbertson et al. (2008). The earlier models for sedimentation from plumes are summarized briefly subsequently.

\section{Plume Model}

The basic ("top-hat") integral model (see Fig. 1) assumes that the flow can be described at any point along the plume path, $S$, by the mean properties of the plume: plume radius, $R$; velocity, $V$; and reduced gravity, $g^{\prime}\left[g^{\prime}=g\left(\rho_{A} \rho_{P}\right) / \rho_{A}\right.$, where $\rho_{A}=$ ambient fluid density and $\rho_{P}=$ mean plume density]. It is assumed that the density contrasts are relatively small, so that the Boussinesq approximation is valid. The entrainment of ambient fluid into the plume is modeled by using an entrainment assumption, where the entrainment velocity is taken to be proportional to the mean plume speed, with ratio $\alpha$ (assumed constant, for simplicity). Thus the volume flow rate in the plume, $Q=\pi R^{2} V$, increases with distance. An important parameter is the jet length, defined here as

$$
L_{J}=\frac{1}{\pi^{1 / 4} \sqrt{2 \alpha}} B_{0}^{-1 / 2} H_{0}^{3 / 4}
$$

where $H_{0}=Q V \cos \theta=$ (specific) horizontal momentum flux; and $B_{0}=Q g^{\prime}=$ buoyancy flux (both conserved quantities in this model) [note the $\sqrt{2}$ factor is erroneously missing from the length scale definition in Lane-Serff et al. (1993)]. The jet length is the distance over which the buoyancy forces begin to dominate and the plume turns toward the vertical. Distances are nondimensionalized with the jet length $\left(x=X / L_{J}, z=Z / L_{J}, s=S / L_{J}\right)$ and the volume flow rate can be nondimensionalized by a characteristic flow rate $\left(q=Q / Q_{J}\right)$ where

$$
Q_{J}=\pi^{1 / 4} \sqrt{2 \alpha} B_{0}^{-1 / 2} H_{0}^{5 / 4}
$$

Writing $\theta$ for the angle between the plume centerline and the horizontal, a pair of equations can be derived for the evolution of the plume along the plume path:

$$
\frac{d(\tan \theta)}{d s}=q \cos \theta
$$
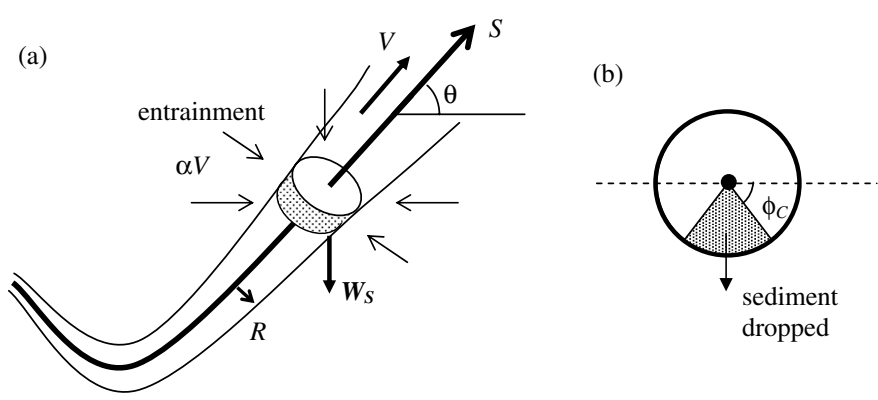

Fig. 1. (a) Sketch illustrating the integral plume model, described in terms of the along-plume coordinate $S$; (b) slice through the plume perpendicular to the plume centerline showing the region in the lower section of the plume from which the sediment falls and the definition of the angle $\phi_{C}$ used in Eq. (14) and

$$
\frac{d q}{d s}=(\cos \theta)^{-1 / 2}
$$

Note that $\cos \theta$ can be written as $\left(1+\tan ^{2} \theta\right)^{-1 / 2}$, so Eqs. (10a) and $(10 b)$ can be treated as a pair of equations in the two variables $q$ and $\tan \theta$. Other flow variables can be derived from $q$ and $\theta$, while the plume centerline is given by

$$
\frac{d X}{d S}=\frac{d x}{d s}=\cos \theta, \quad \frac{d Z}{d S}=\frac{d z}{d s}=\sin \theta
$$

For convenience the equations are usually integrated from a "virtual origin," where $q=0$ and $\theta=\theta_{0}$.

\section{Noncohesive Sediment}

For the normal, noncohesive sediment model, it is assumed that the sediment has a uniform fixed fall speed $W_{S}$ and is sufficiently dilute as to have a negligible effect on the plume density (so the Boussinesq approximation still holds). In this model (Lane-Serff and Moran 2005) the sediment drops out of the plume where the component of the fall velocity perpendicular to the plume boundary exceeds the entrainment velocity (Fig. 1). A scale for the plume velocity is given by

$$
V_{J}=\sqrt{2 \alpha} \pi^{-1 / 4} B_{0}^{1 / 2} H_{0}^{-1 / 4}
$$

and this can be used to give a nondimensionalized version of the fall speed

$$
w_{S}=W_{S} / V_{J}
$$

Lane-Serff and Moran (2005) show that this nondimensional fall speed is the only extra parameter required (in addition to those for the basic plume) to characterize the distribution of sediment falling from the plume. In addition to the plume equations, an equation is derived for the nondimensional sediment load, $p$ (where $p=P / P_{0}$, so $p=1$ at the source)

$$
\frac{d p}{d s}=-\left(\frac{2 w_{S}}{\pi}\right) p(\cos \theta)^{1 / 2}\left[\cos \theta \cos \phi_{C}-\frac{\left(\pi / 2-\phi_{C}\right)}{2 w_{S} q \cos \theta}\right]
$$

(provided $2 w_{s} q \cos ^{2} \theta>1$, else $d p / d s=0$ ), where

$$
\sin \phi_{C}=1 /\left(2 w_{s} q \cos ^{2} \theta\right)
$$

Here $\phi_{C}$ marks the region in the lower part of the plume from which the sediment is able to drop through the plume boundary (see Fig. 1).

Once the plume is rising vertically, it will continue to rise until it reaches the free surface (or possibly a neutral level in a stratified ambient fluid) and spreads as a gravity current. In principle there could be further deposition from the rising plume once the plume velocity drops to around the sediment fall speed. However, the velocity in a vertical buoyant plume drops relatively slowly $\left(\sim z^{1 / 3}\right)$ so that in practice there is unlikely to be any further deposition until the plume reaches the free surface or a neutral density level and begins to spread horizontally. Thus the load carried by the rising plume reaches a steady nonzero value [see Lane-Serff and Moran (2005) for further discussion of this point]. Lane-Serff and Moran (2005) and Mugford and Lane-Serff (2007) deal with deposition from both the plume and the gravity current part of the flow and achieve good agreement for the distribution of sediment with some laboratory experiments. Any sediment falling from the rising plume, and some sediment falling from the spreading current, will be reentrained by the plume (e.g., German and Sparks 1993; 
Lane-Serff 1995). The deposition from gravity currents in general is considered subsequently.

\section{Cohesive Sediments}

For cohesive sediments the (mean) fall speed is no longer fixed but tends toward an equilibrium value given by Eq. $(6 a)$. To use this an expression is needed for the dissipation rate, $\varepsilon$, in terms of the bulk flow properties. Lane-Serff (1993), for example, shows that the mean flow has a dissipation rate given by

$$
\varepsilon=\alpha \frac{V^{3}}{R}
$$

Making use of Eq. (6a), a general expression for the equilibrium fall speed can be written as

$$
W_{S e}=W_{S 0} \frac{C}{C_{0}}\left(\frac{\varepsilon_{0}}{\varepsilon}\right)^{1 / 4}=W_{S 0} \frac{C}{C_{0}}\left(\frac{V_{0}}{V}\right)^{3 / 4}\left(\frac{R}{R_{0}}\right)^{1 / 4}
$$

where $W_{S 0}=$ equilibrium fall speed when the sediment concentration is $C_{0}$, the plume radius $R_{0}$ and the plume speed $V_{0}$. This can be rewritten in terms of the total sediment load, $P=Q C$, and the total momentum flux, $M=Q V$, as follows:

$$
W_{S e}=W_{S 0} \frac{P}{P_{0}}\left(\frac{M}{M_{0}}\right)^{-7 / 8}
$$

or, in nondimensional terms, as

$$
w_{S e}=w_{S 0} p\left(\frac{\cos \theta}{\cos \theta_{0}}\right)^{7 / 8}
$$

A useful feature of this equation is that the equilibrium fall speed does not depend directly on the flow rate in the plume and has a finite value $\left(w_{S 0}\right)$ at the virtual origin. In the initial jet part of the flow (where the momentum flux and angle are changing slowly), if there is little or no deposition, then the sediment load remains constant, and thus so does the equilibrium fall speed. Furthermore, as one approaches the virtual origin the time scales for the aggregation and break-up processes become zero, from Eq. (2b), indicating that the equilibrium floc size and fall speed would be realized (at least for a virtual origin).

\section{Equilibrium Model}

Assuming that the floc adjustment time scale $T_{A}$ is always small, then a simple model can be constructed for a flocculating sediment in a buoyant plume by assuming that the fall speed is always equal to the local equilibrium value. For this model, the introduction of a cohesive sediment has not increased the number of parameters compared with the noncohesive model, with the nondimensional fall speed $\left(w_{S}\right)$ now replaced by the nondimensional fall speed at the virtual origin $\left(w_{S 0}\right)$. The differential equations remain the same, but the $w_{S}$ in Eqs. $(14 a)$ and $(14 b)$, which was a constant for the noncohesive model, is now a variable given by Eq. (17b). The equations can be integrated numerically from a virtual origin (where $q=0$ and $p=1$ ) for various values of the parameters $w_{S 0}$ and $\theta_{0}$. Even for cases where $T_{A}$ is significant, the simple model provides a useful bound because the real results (with a floc size intermediate between the initial size and the local equilibrium value) will lie somewhere between the results obtained by using a noncohesive model (with the floc fixed at its initial size) and results obtained using the equilibrium floc size model (with the flocs instantaneously adjusting to their equilibrium size).

\section{Nonequilibrium Model}

Using the approximate unsteady floc size Eq. (7a) and assuming the flocs move with the mean flow speed in the plume, the following can be written:

$$
\frac{d D}{d S} \approx-\frac{D^{\prime}}{V T_{A}}
$$

After some manipulation, this can be used to obtain an equation for the nondimensional fall speed

$$
\frac{d w_{S}}{d s}=-B \cos \theta \frac{p^{3}}{q^{2}} \frac{w_{S}-w_{S e}}{w_{S e}}
$$

where $w_{S e}=$ nondimensional equilibrium fall speed given by Eq. (17b); and $B=$ constant given by

$$
B=\frac{1}{4}\left(\cos \theta_{0}\right)^{3 / 4} W_{S 0} \pi^{-3 / 8} \alpha^{-9 / 4} \nu^{-1 / 4} k_{A}^{2} k_{B}^{-1} P_{0}^{2} M_{0}^{-13 / 8}
$$

where $P_{0}=$ initial sediment load $\left(\mathrm{kg} \mathrm{m}^{-3}\right)$; and $M_{0}=V_{0} Q_{0}=$ initial specific momentum flux $\left(\mathrm{m}^{4} \mathrm{~s}^{-2}\right)$. These equations show that the fall speed tends toward the equilibrium fall speed at a local rate that depends on the constant $B$. It can be shown that $B \sim w_{S 0} T_{J} / T_{A J}$, where $T_{J}=L_{J} / V_{J}=$ natural time scale for the plume (the time it takes to move a jet length when moving at the characteristic velocity scale $V_{J}$ ) and $T_{A J}=$ floc adjustment time scale (based on the concentration, speed, and turbulence in the plume a jet length from the source). If the adjustment time scale is relatively small, then $B$ is large and the fall speed is close to the equilibrium value, whereas if the adjustment time scale is large, then the adjustment to the local equilibrium value is slow. However, $B$ is the time scale ratio a jet length from the source: the local adjustment rate also depends on the ratio $\left(p^{3} / q^{2}\right)$, which tends to infinity as one approaches a virtual origin (where $q=0$ ) and gets smaller as the volume flux increases and sediment load decreases along the plume.

\section{Results for Turbulent Plumes}

\section{Results of the Equilibrium Model}

This section first reviews the results from the equilibrium model. Examples of the distribution of sediment dropping from the plume are shown in Fig. 2. Sediment typically drops from the plume within a few jet-lengths of the source, where the plume is close to horizontal. Once the plume is rising near vertically, there is

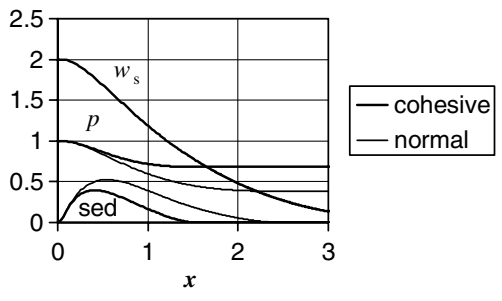

(a)

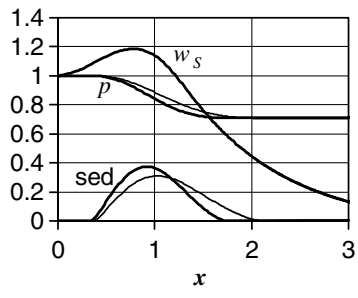

(b)
Fig. 2. Equilibrium model results for plume flows, showing sediment deposition (sed), sediment load in the plume ( $p$ ), and (for the cohesive sediment) the fall speed $\left(w_{S}\right)$ as functions of the horizontal distance, $x$; conditions at the source are (a) $w_{S 0}=2.0, \theta_{0}=0^{\circ}$ (horizontal); (b) $w_{S 0}=1.0, \theta_{0}=-45^{\circ}$ (downward); for normal sediment the fall speed remains constant at its initial value 


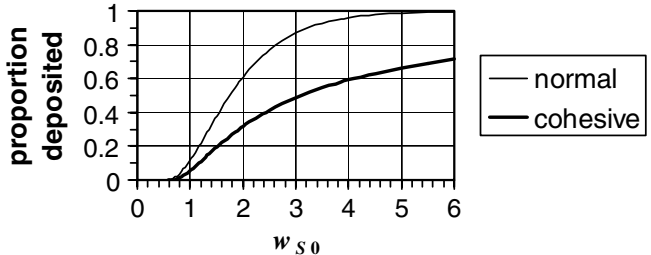

Fig. 3. Total fraction of the initial sediment load deposited from turbulent plumes injected horizontally as a function of the initial fall speed $w_{S 0}$ (equilibrium model)

assumed to be no further sediment deposition (in practice, any further deposition is likely to occur once the flow has reached the free surface or a level of neutral density and begun spreading horizontally-see preceding discussion).

For the cohesive sediment, the fall speed decreases as the sediment load decreases and the momentum flux increases. For a plume injected horizontally [e.g., Fig. 2(a)] the fall speed decreases monotonically from the source. However, for a plume injected downward [e.g., Fig. 2(b)], the plume initially decelerates, and the momentum flux reduces until the plume turns and begins to rise. In this case it is possible for the equilibrium fall speed to increase initially, giving larger sedimentation rates at the lowest point of the plume trajectory.

For horizontal sources, however, the fall speed is always lower for the cohesive sediment than for the corresponding flow with a fixed fall speed. This is because the equilibrium floc size reduces away from the source: both the aggregation and break-up processes are diminished as the turbulence reduces away from the source, but the aggregation process is also affected by the reduced concentration of sediment. As a result, the proportion of cohesive sediment deposited by the plume is always less than the corresponding noncohesive case (see Fig. 3). It is important to note that "noncohesive" here means flocs that remain at their initial size and do not break up to form smaller flocs. If the sediment consisted entirely of the basic particles that made up the flocs, then the fall speed would be much smaller and the deposition would be much weaker, or possibly zero.

\section{Results of the Nonequilibrium Model}

This section presents results where the (mean) fall speed is not always equal to the local equilibrium value. This introduces the new parameter, $B$, which depends on source conditions, and the fluid and floc properties [Eq. (19b)], with $B=0$ representing a noncohesive sediment (with fall speed fixed to its initial value) and $B=\infty$ corresponding to the equilibrium model (fall speed adjusts instantly to the local equilibrium value).

Examples of model results for horizontal sources are shown in Fig. 4. For the case shown (initial nondimensional fall

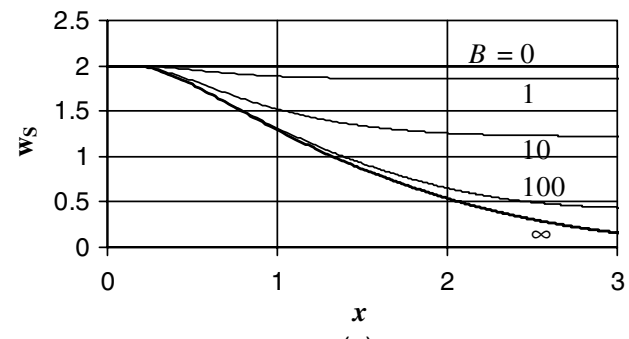

(a) speed $w_{S 0}=2$ ), a value of the time scale ratio of $B=1$ results in only a small change in the fall speed from its initial value and a sediment distribution that is close to that for the simpler model with no change in fall speed. Conversely, when $B=100$ the resulting distribution is close to that given by the equilibrium model because the floc size now adjusts very rapidly to be close to the equilibrium value (at least for the part of the flow within a few jet lengths of the source, which is where the deposition takes place). For $B=10$, the fall speed and sediment distribution lies between the two simpler models, closer to the equilibrium model near the source (because the adjustment time scale is always short there) and moving toward a fixed fall speed further from the source (as the time scale increases).

\section{Deposition from Gravity Currents and Turbidity Currents}

This section considers the application of the equilibrium floc size model to two other types of buoyancy-driven flows: gravity currents and turbidity currents. In both cases two-dimensional and axisymmetric forms are considered. For the gravity current flows, where the sediment is carried passively, explicit functions are found for the concentration and sedimentation rates. These are different in form from the noncohesive equations, with an increased spread of the cohesive sediment as the fall speed decreases with decreasing concentration, but the quantitative differences are small. In the turbidity current models, the primary change from the noncohesive sediment is that the currents with cohesive sediment continue (in principle) indefinitely, whereas the noncohesive sediment turbidity currents flow to a finite distance from the source. In practice, however, the fall speed would have a limiting minimum value (not included in the present model for simplicity), so that a finite range for cohesive sediment would also be expected.

\section{Models for Gravity Currents}

It is assumed that the flow is driven by a constant source flux of particle-laden dense fluid and that the sedimenting particles are sufficiently dilute as to have a negligible effect on the fluid density. For these flows, it is assumed that the turbulent scales are again related to the larger scales, so that the turbulent dissipation is related to the local flow parameters by

$$
\varepsilon \propto \frac{U^{3}}{D}
$$

where $U=$ gravity current speed; and $D=$ gravity current depth. Note that the only requirement is that the relations between the global and turbulent velocity and length scales are approximately

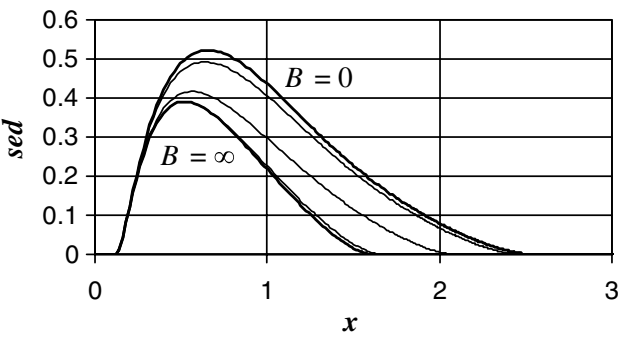

(b)

Fig. 4. (a) Nondimensional floc fall speed; (b) sediment deposition from buoyant plumes injected horizontally with initial nondimensional fall speed $w_{S 0}=2$ and various values of the parameter $B(0,1,10,100, \infty)$ 
constant for a particular flow because it is only the relative size of the dissipation rate that is used in calculating the fall speed.

\section{Two-Dimensional Gravity Currents}

For two-dimensional gravity currents, the depth and speed are constant with distance $X$, so $U=U_{0}$ and $D=D_{0}$. As a result the dissipation rate is also constant, so that the change in fall speed of the cohesive sediment comes only from the change in the sediment concentration $C$. Thus, if the fall speed at $X=0$ (where the concentration is $C_{0}$ ) is $W_{S 0}$, then

$$
W_{S}=W_{S 0} C / C_{0}
$$

Deposition of sediment from the current is given by

$$
\frac{d\left(U_{0} D_{0} C\right)}{d X}=-W_{S} C=-W_{S 0} C^{2} / C_{0}
$$

Nondimensionalizing the concentration and horizontal distance as follows

$$
c=C / C_{0} \quad \text { and } \quad x=X / X_{S}, \quad \text { where } X_{S}=\left(U_{0} D_{0}\right) / W_{S 0}
$$

and integrating Eq. (22), the concentration of sediment in the current is given by

$$
c=1 /(1+x)
$$

and the rate of sedimentation is given by

$$
\text { sed }=1 /(1+x)^{2}
$$

These equations are plotted in Fig. 5.

For comparison, the corresponding concentration and sedimentation rate distributions for noncohesive sediment are both of the form $\exp (-x)$. The concentration of sediment decays more slowly for the cohesive sediment, as the fall speed gradually decreases from its initial value. As a result, the deposition rate is lower near the origin (compared to noncohesive sediment) but is higher further from the source (as more sediment remains in the current for longer distances).

\section{Axisymmetric Gravity Currents}

For axisymmetric gravity currents carrying noncohesive sediment, the concentration and sedimentation rate both have the Gaussian form $\exp \left(-\pi r^{2}\right)$, where $r=R / R_{S N}$ and $R_{S N}=\sqrt{ }\left(Q / W_{S 0}\right)$ (see Sparks et al. 1991). However, when cohesive sediment in axisymmetric gravity currents is considered, some assumptions are needed about the form of the flow. There are two solutions to the steady, axisymmetric gravity current equations: a supercritical solution, with the speed tending to a constant value and the height $D \sim$ $1 / R$ as $R \rightarrow \infty$; and a subcritical solution, with $D$ tending to a

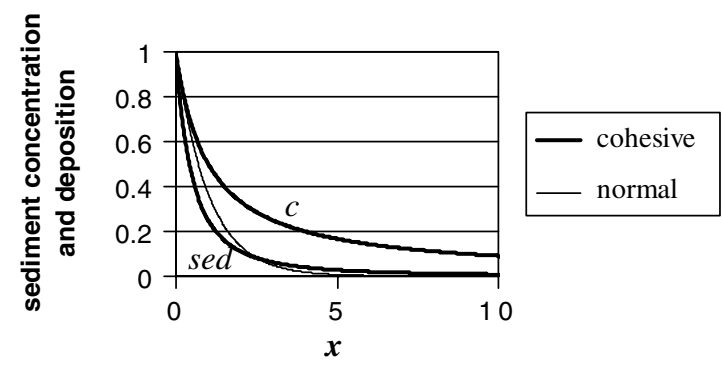

Fig. 5. Sediment concentration (c) and deposition rate (sed) for a twodimensional gravity current; for noncohesive sediment, the (nondimensional) concentration and sedimentation rate curves are equal constant value $\left(D_{0}\right.$, say) and $V \sim 1 / R$ as $R \rightarrow \infty$. In practice, the supercritical solution is unstable and is likely to be associated with mixing and a hydraulic jump to the subcritical flow, so the subcritical flow is used as the basis for subsequent analysis.

For axisymmetric gravity currents there is no simple (nonzero) value of the fall speed at the origin that could be used for defining the fall speed elsewhere in the flow. Instead, it is assumed that the fall speed is some known value $W_{S 0}$, when $C=C_{0}, D=D_{0}$ and $R=R_{0}$ [and thus $\left.V=V_{0}=Q /\left(2 \pi R_{0} D_{0}\right)\right]$. Assuming the dissipation rate has the same form as the two-dimensional case given by Eq. (20) and again using Eq. (6a), this gives an equation for the fall speed:

$$
W_{S}=W_{S 0}\left(\frac{R}{R_{0}}\right)^{3 / 4} \frac{C}{C_{0}}
$$

The equation for deposition of sediment is

$$
\frac{d(Q C)}{d R}=-2 \pi W_{S} C
$$

The natural scale for nondimensionalizing distances, $R_{S}$, is a combination of $R_{0}$ and $R_{S N}$ (the scale for the noncohesive sediment) with

$$
R_{S}=R_{0}^{3 / 7} R_{S N}^{4 / 7}
$$

Writing $c=C / C_{0}$ and $r=R / R_{S}$ and integrating Eq. (26) gives the concentration in the current as

$$
c=\left(1+\frac{8 \pi}{11} r^{11 / 4}\right)^{-1}
$$

and the sedimentation rate as

$$
\operatorname{sed}=r^{3 / 4}\left(1+\frac{8 \pi}{11} r^{11 / 4}\right)^{-2}
$$

These equations are plotted in Fig. 6.

The sedimentation rate (and fall speed) tends to zero as $r$ tends to zero, which is why there is no suitable scale for the fall speed at the origin. The sedimentation rate reaches a maximum value at $r=0.38$, and the sediment concentration decays more slowly than for the noncohesive case (although the length scales are not identical in the two cases).

\section{Models for Turbidity Currents}

This section considers turbidity currents generated by the release of a finite volume of fluid containing particles in suspension. Considered here for simplicity is the case in which the fluid in the current has the same density as the ambient fluid so that the density contrast is entirely attributable to the concentration of sediment. Thus the

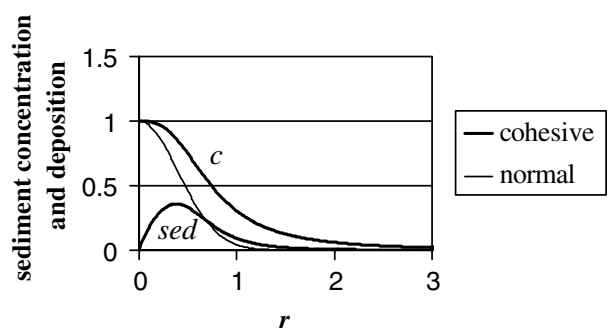

Fig. 6. Sediment concentration (c) and deposition rate (sed) for am axisymmetric gravity current; for noncohesive sediment, the (nondimensional) concentration and sedimentation rate curves are equal 
reduced gravity is given by $g^{\prime}=g_{0}^{\prime}\left(C / C_{0}\right)$. The flow is represented by a simple box model, with height at any instant $D$, and speed, $U$, given by some Froude number dependence on the height:

$$
U=\mathrm{F} \sqrt{\left(g^{\prime} D\right)}
$$

Applications of this type of model, and more complicated models, to turbidity currents with noncohesive sediment are given by, for example, Bonnecaze et al. (1995) and Dade and Huppert (1995). The same relation between dissipation rate and flow properties as for the gravity currents is assumed, given by Eq. (20).

\section{Two-Dimensional Turbidity Currents}

The finite release is assumed to have a fixed cross-sectional area $A_{0}=D L$, where $L=$ length of the turbidity current (i.e., no entrainment into the current). As for the axisymmetric gravity currents, it is necessary to use a representative value for the fall speed for conditions other than at the origin. Here it is assumed that the fall speed is some known value $W_{S 0}$ when $C=C_{0}$ and $L=L_{0}$, and thus $D=D_{0}=A_{0} / L_{0}$ and $U_{0}=\mathrm{F} \sqrt{\left(g^{\prime} D\right)}$. Using Eq. (6a), this gives the general fall speed as

$$
W_{S}=W_{S 0}\left(\frac{D}{D_{0}}\right)^{-1 / 8}\left(\frac{C}{C_{0}}\right)^{5 / 8}
$$

It is then possible to derive a pair of coupled equations for the length and concentration in terms of the time, $T$ :

$$
\frac{d L}{d T}=\mathrm{F} \sqrt{g^{\prime} D} \quad \text { and } \quad \frac{d\left(C A_{0}\right)}{d T}=-W_{S} C L
$$

The length and time scales $\left(L_{S}\right.$ and $\left.T_{S}\right)$ can be related to those appropriate to a two-dimensional turbidity current with noncohesive sediment $\left(L_{S N}\right.$ and $\left.T_{S N}\right)$ as follows:

$$
\begin{aligned}
& L_{S}=\left(\mathrm{F}_{0}^{1 / 2} A_{0}^{3 / 2} L_{0}^{1 / 8} W_{S 0}^{-1}\right)^{8 / 21}=L_{S N}^{20 / 21} L_{0}^{1 / 21} \text { and } \\
& T_{S}=\left(\mathrm{F}^{-6} g_{0}^{\prime-3} A_{0}^{5} L_{0} W_{S 0}^{-8}\right)^{1 / 14}=T_{S N}\left(L_{0} / L_{S N}\right)^{1 / 14}
\end{aligned}
$$

Using these scales to nondimensionalize the equations (so $l=L / L_{S}, t=T / T_{S}$, etc) gives

$$
\frac{d l}{d t}=c^{1 / 2} l^{-1 / 2} \quad \text { and } \quad \frac{d c}{d t}=-c^{13 / 8} l^{9 / 8}
$$

Integrating these from a virtual origin where $t=0, l=l_{0} \approx 0$ and $c=1$, we find a relation between $c$ and $l$ :

$$
c=\left(1+\frac{1}{21} l^{21 / 8}\right)^{-8}
$$

which can be used to find implicit equations relating $c$ and $l$ to $t$. The sedimentation rate at any position in the current at any instant is the same, so that the total deposition at position $l$ is given by integrating the sediment flux from the time the current passes position $l\left(t=t_{l}\right)$ :

$$
\operatorname{dep}(l)=\int_{t=t_{l}}^{\infty} w_{S} c d t=\int_{l^{\prime}=l}^{\infty} c^{9 / 8} l^{15 / 8} d l^{\prime}
$$

The main results are plotted in Fig. 7. For normal turbidity currents (with noncohesive sediment), the current travels a finite distance (e.g., Bonnecaze et al. 1995), whereas for cohesive sediment the fall speed decreases as $c$ decreases so that the current continues indefinitely. In reality there is a nonzero minimum fall speed (given by the fall speed of the individual particles making up the flocs) so that the turbidity current would not continue indefinitely. Even for the cohesive sediment model used here, the bulk of the sediment is deposited within a distance of twice the length scale, $L_{S}$.

\section{Axisymmetric Turbidity Currents}

For axisymmetric turbidity currents the box model represents the current as a having a cylindrical shape with fixed volume $V_{0}=\pi R^{2} D$, where $R=$ radius of the current. Again it is assumed that the fall speed is some known value $W_{S 0}$ when $C=C_{0}$ and $R=R_{0}$, and thus $D=D_{0}=V_{0} /\left(\pi R_{0}^{2}\right)$ and $U_{0}=\mathrm{F} \sqrt{\left(g_{0}^{\prime} D_{0}\right)}$. Using Eq. (6a) gives the same general fall speed (in terms of $C$ and $D$ ) as given in Eq. (27). The coupled equations for the axisymmetric turbidity current are

$$
\frac{d R}{d T}=\mathrm{F} \sqrt{g^{\prime} D} \quad \text { and } \quad \frac{d\left(C V_{0}\right)}{d T}=-W_{S} C \pi R^{2}
$$

The length and time scales $\left(R_{S}\right.$ and $\left.T_{S}\right)$ can again be related to those appropriate to the noncohesive axisymmetric turbidity current $\left(R_{S N}\right.$ and $\left.T_{S N}\right)$ as follows:

$R_{S}=\left[\mathrm{F}^{4} g_{0}^{\prime 2}\left(V_{0} / \pi\right)^{6} R_{0} W_{S 0}^{-4}\right]^{1 / 17}=R_{S N}^{16 / 17} R_{0}^{1 / 17}$ and

$T_{S}=\left[\mathrm{F}^{-18} g_{0}^{\prime-9}\left(V_{0} / \pi\right)^{7} R_{0}^{4} W_{S 0}^{-16}\right]^{1 / 34}=T_{S N}\left(R_{0} / R_{S N}\right)^{2 / 17}$

Using these scales to nondimensionalize the equations (e.g., $\left.r=R / R_{S}, t=T / T_{S}\right)$ gives

$$
\frac{d r}{d t}=c^{1 / 2} r^{-1} \quad \text { and } \quad \frac{d c}{d t}=-c^{13 / 8} r^{9 / 4}
$$

Integrating these from a virtual origin where $t=0, r=r_{0} \approx 0$, and $c=1$ results in a relation between $c$ and $r$ :

$$
c=\left(1+\frac{1}{34} r^{17 / 4}\right)^{-8}
$$

which can be used to find implicit equations relating $c$ and $r$ to $t$. The total deposition at a given radial position $r$ is found using a similar integral to the two-dimensional case. The results are plotted

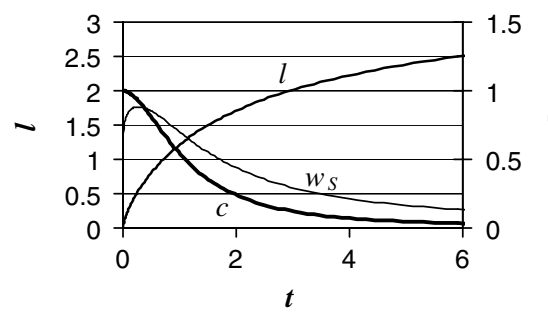

(a)

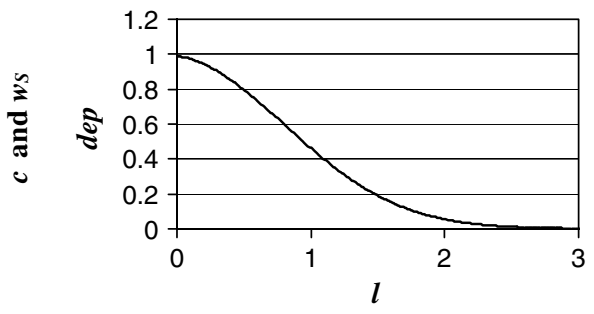

(b)

Fig. 7. Model results for two-dimensional turbidity currents with cohesive sediment: (a) length, sediment concentration, and fall speed of the current as functions of time; (b) total deposition from the current as a function of position 


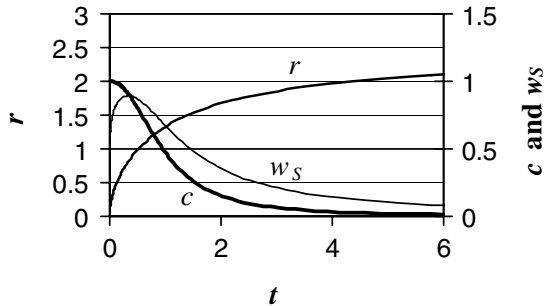

(a)

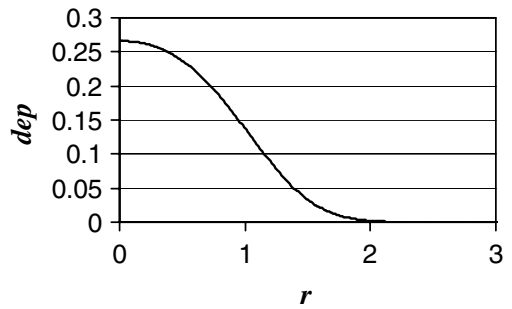

(b)

Fig. 8. Model results for axisymmetric turbidity currents with cohesive sediment: (a) radius, sediment concentration, and fall speed of the current as functions of time; (b) total deposition from the current as a function of radial position

in Fig. 8. Again the cohesive sediment (with the fall speed decreasing at large times) continues indefinitely, but with the bulk of the sediment deposited within $2 R_{S}$ of the origin.

\section{Discussion and Conclusions}

This paper demonstrates how cohesive sediment can be incorporated into models of turbulent plumes, gravity currents, and turbidity currents. The model for the cohesive sediment assumes that the fall speed is known for some standard flow conditions. In principle, flocculation models (e.g., Winterwerp 1998) give the fall speed in terms of flow conditions and a number of constants relating to the properties of the fluid and the sediment particles (such as surface chemistry). In practice, however, these quantities are estimated by carrying out a series of experiments covering a range of flow properties, measuring the fall speed, and inferring the values of the underlying constants from the flocculation model. Thus assuming that the fall speed is known for some flow conditions and using the flocculation model to give the functional form for the dependence of the equilibrium fall speed as the flow properties change (as is done here) is a reasonable and practical approach. The end result is an equilibrium floc size that is proportional to the sediment concentration and the Kolmogorov length scale.

For plumes the useful result was found that the fall speed is well-defined at the virtual origin and also changes only slowly in the early part of the plume. Thus the same number of parameters is needed to describe the cohesive case as the noncohesive case, if one can assume that the fall speed is always close to the equilibrium value. For cohesive sediment the fall speed parameter is now the nondimensional fall speed at the virtual origin. For practical applications, this can be written in terms of the source conditions as

$$
w_{S 0}=W_{S 0} / V_{J}=W_{S 0}\left(\cos \theta_{0}\right)^{1 / 4}\left(2 \alpha g_{0}^{\prime} R_{0}\right)^{-1 / 2}
$$

For most plumes flows, the fall speed for the cohesive sediment drops below its initial value, so that the sedimentation rates are smaller than for the noncohesive sediment. However, for downward-directed plumes, the flow speed and thus the dissipation rate reduce, so that it is possible for the flocs and their fall speed to get larger than their initial values.

In considering plume flows where the adjustment to the equilibrium floc size cannot be regarded as instantaneous, a new parameter $(B)$ is introduced, which can be related to the ratio of a typical plume time scale to a typical flocculation time scale.

For plumes injected horizontally, the results of the nonequilibrium model generally lie between the noncohesive and equilibrium results, so these simpler models provide useful bounds. Although the parameter $B$ captures the typical behavior (at around a jet length from the source, where the bulk of sedimentation occurs), the local adjustment time scale always tends to zero toward the source (so that an equilibrium model is always valid approaching the virtual origin), and the adjustment time scale always increases downstream, so that eventually the floc size remains approximately constant (although this may be beyond the point where sedimentation has ceased anyway).

The equilibrium model was also applied to gravity currents and turbidity currents. The nonequilibrium model was not developed for these cases, but based on plume results, it is generally expected that the results of a nonequilibrium model will lie between the equilibrium model results (assuming instant adjustment of floc size to the local conditions) and the fixed fall speed results. The functional forms of the sediment concentrations and deposition patterns are identified, along with the relevant length scales.

In the models presented here, the fractal dimension of the flocs was taken to be $n_{f}=2$. As noted previously, the equilibrium floc size does not depend on $n_{f}$, at least to a first approximation. However, the fall speed does depend on the fractal dimension. This could be incorporated into all the models described in this paper: the differences would appear in the fractional powers in the various equations and the resulting distributions, but the basic analysis would follow the same pattern.

\section{Acknowledgments}

This work was partly supported by EPSRC Grant GR/S25128/01. Thanks also to the reviewers and editor for suggesting various improvements to the manuscript.

\section{References}

Bonnecaze, R. T., Hallworth, M. A., Huppert, H. E., and Lister, J. R. (1995). "Axisymmetrical particle-driven gravity currents." J. Fluid Mech., 294, 93-121.

Cuthbertson, A. J. S., Apsley, D. D., Davies, P. A., Lipari, G., and Stansby, P. K. (2008). "Deposition from particle-laden, plane, turbulent, buoyant jets." J. Hydraul. Eng., 134(8), 1110-1122.

Cuthbertson, A. J. S., and Davies, P. A. (2008). "Deposition from particleladen, round, turbulent, horizontal, buoyant jets in stationary and coflowing receiving fluids." J. Hydraul. Eng., 134(4), 390-402.

Dade, W. B., and Huppert, H. E. (1995). "A box model for non-entraining, suspension-driven gravity surges on horizontal surfaces." Sedimentology, 42(3), 453-471.

German, C. R., and Sparks, R. S. J. (1993). "Particle recycling in the TAG hydrothermal plume.” Earth Planet. Sci. Lett., 116(1-4), 129-134.

Jarvis, P., Jefferson, B., Gregory, J., and Parsons, S. A. (2005). "A review of floc strength and breakage." Water Res., 39(14), 3121-3137.

Lane-Serff, G. F. (1993). "Investigation of the fractal structure of jets and plumes." J. Fluid Mech., 249, 521-534.

Lane-Serff, G. F. (1995). "Particle recycling in hydrothermal plumes: Comment on particle recycling in the TAG hydrothermal plume by C. R. German and R. S. J. Sparks.” Earth Planet. Sci. Lett., 132(1-4), 233-234. 
Lane-Serff, G. F., Linden, P. F., and Hillel, M. (1993). "Forced, angled plumes." J. Hazard. Mat., 33(1), 75-99.

Lane-Serff, G. F., and Moran, T. J. (2005). "Sedimentation from buoyant jets." J. Hydraul. Eng., 131(3), 166-174.

Mugford, R., and Lane-Serff, G. F. (2007). "Sedimentation from buoyant jets: Errata." J. Hydraul. Eng., 133(4), 462.

Sparks, R. S. J., Carey, S. N., and Sigurdsson, H. (1991). "Sedimentation from gravity currents generated by turbulent plumes." Sedimentology, 38(5), 839-856.

Thomas, D. N., Judd, S. J., and Fawcett, N. (1999). "Flocculation modelling: A review." Water Res., 33(7), 1579-1592.

Winterwerp, J. C. (1998). "A simple model for turbulence induced flocculation of cohesive sediment." J. Hydraul. Res., 36(3), 309-326. 